\title{
Imaging of Hereditary Hemorrhagic Telangiectasia
}

\author{
Marie-France Carette $\cdot$ Cosmina Nedelcu $\cdot$ Marc Tassart • \\ Jean-Didier Grange $\cdot$ Marie Wislez $\cdot$ Antoine Khalil
}

Received: 4 December 2007/ Accepted: 3 March 2008/Published online: 30 October 2008

(C) Springer Science+Business Media, LLC 2008. This article is published with open access at Springerlink.com

\begin{abstract}
This pictorial review is based on our experience of the follow-up of 120 patients at our multidisciplinary center for hereditary hemorrhagic telangiectasia (HHT). Rendu-Osler-Weber disease or HHT is a multiorgan autosomal dominant disorder with high penetrance, characterized by epistaxis, mucocutaneous telangiectasis, and visceral arteriovenous malformations (AVMs). The research on gene mutations is fundamental and family screening by clinical examination, chest X-ray, research of pulmonary shunting, and abdominal color Doppler sonography is absolutely necessary. The angioarchitecture of pulmonary AVMs can be studied by unenhanced multidetector computed tomography; however, all other explorations of liver, digestive bowels, or brain require
\end{abstract}

Electronic supplementary material The online version of this article (doi:10.1007/s00270-008-9344-2) contains supplementary material, which is available to authorized users.

M.-F. Carette $(\varangle) \cdot$ C. Nedelcu · M. Tassart · A. Khalil

Radiology Department, AP-HP Tenon Hospital,

4 Rue de la Chine, 75020 Paris, France

e-mail: marie-france.carette@tnn.aphp.fr

M.-F. Carette

Pierre et Marie Curie University, Paris VI, France

M.-F. Carette · C. Nedelcu · M. Tassart · J.-D. Grange ·

M. Wislez · A. Khalil

Centre d'Accueil de la Maladie de Rendu Osler de Tenon (CAMROT), Paris, France

e-mail: antoine_khalil@yahoo.fr

\section{J.-D. Grange}

Hepatogastroenterology Department, AP-HP Tenon Hospital, Paris, France

M. Wislez

Department of Pneumology, AP-HP Tenon Hospital, Paris,

France administration of contrast media. Magnetic resonance angiography is helpful for central nervous system screening, in particular for the spinal cord, but also for pulmonary, hepatic, and pelvic AVMs. Knowledge of the multiorgan involvement of HHT, mechanism of complications, and radiologic findings is fundamental for the correct management of these patients.

Keywords Rendu-Osler Weber disease .

Pulmonary arteriovenous malformation - Genetic disease .

Hepatic arteriovenous malformation .

Pancreatic telangiestasia - Brain abscess .

Right-to-left shunt · Transcatheter occlusion · Reperfusion · Hereditary hemorrhagic telangiectasia

\section{Introduction}

Hereditary hemorrhagic telangiectasia (HHT), or RenduOsler-Weber disease, is an autosomal dominant multiorgan pathology with a prevalence of 1 in 10,000 to 1 in 5000 [1] and characterized by the presence of multiple small telangiectases of the skin, mucous membranes, gastrointestinal tract, and other organs, with associated recurrent episodes of bleeding from affected sites. Although everyday symptoms in these patients are dominated by epistaxis that may markedly alter their quality of life, more severe manifestations of the disease are due to the consequences of pulmonary arteriovenous malformations (PAVMs) and, less often, to liver, brain, or gastrointestinal tract involvement [1]. The diagnosis of HHT requires the presence of at least three of the following Curacao criteria: recurrent spontaneous epistaxis, mucocutaneous telangiectasis, an 


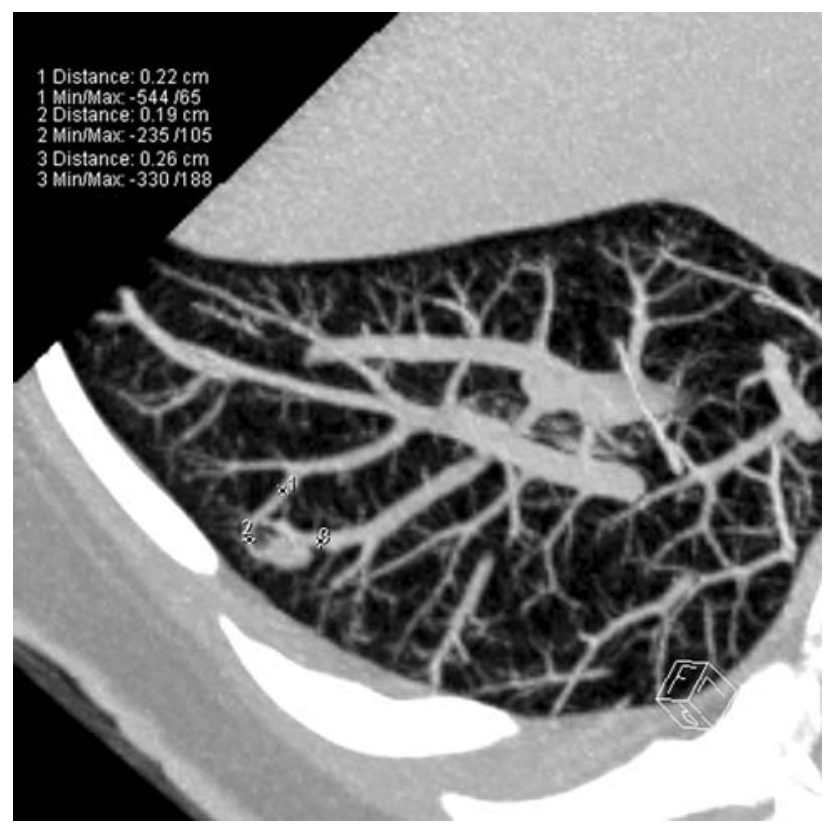

Fig. 1 A 20-year-old woman without a personal or family history fulfilling the Curacao criteria was admitted for cerebral transient ischemic attack; after complete clinical and paraclinical investigations the only abnormality found was paradoxical emboli through a small pulmonary arteriovenous malformation (PAVM). Maximal intensity projection MDCT showing the feeding artery (2), the aneurism, and the draining vein of a simple unique lower right lobe PAVM. The feeding artery is smaller than $3 \mathrm{~mm}$; nevertheless, it had a clinical impact

autosomal dominant familial distribution of the disease, and visceral AVMs.

The mutations of the endoglin gene $(E N G)$ on the $9 \mathrm{q}$ chromosome and the aktivine gene $(A L K l)$ on the $12 \mathrm{q}$ chromosome are implicated in the inheritance of this disease [2]. Furthermore, the $E N G$ mutations determine the HHT1 disease subtype, which correlates with a more critical pulmonary involvement, and $A K L 1$ mutations indicate the HHT2 subtype, with a predominance of liver involvement [2].

The purpose of this article is to provide a brief review of the visceral involvement and the relevant findings on diagnostic and therapeutic imaging.

\section{Lung}

PAVM is the most frequent lesion of the lung in patients with HHT. It occurs in $20 \%$ to $50 \%$ and provokes different clinical symptoms, such as dyspnea, cyanosis, massive hemoptysis, and hemothorax [1]. The shunt induces various paradoxical emboli such as cruoric, bacterial, and gaseous [1]. Currently, it is known that for clinical relevance, the diameter of the artery of the PAVM must be $\geq 3 \mathrm{~mm}$. However, one case with a feeding artery diameter $<3 \mathrm{~mm}$ (Fig. 1) and responsible for ischemic stroke has been reported [3].
The anatomic structure of the PAVM is classified according to the two types [4] defined as simple, with a single artery feeding into an aneurysmal communication with a single draining vein (Fig. 2, Movies 1 and 2), and complex, with two or more arterial branches communicating via an aneurysm with two or more draining veins (Fig. 3). On chest X-ray, a PAVM classically appears as a well-defined nodule with a minimum of two branching vessels (Fig. 4A). However, chest X-ray, although rather specific, is not sensitive [5]. Diagnosis is performed on unenhanced helical CT with a small slab of maximum intensity projection reconstruction showing one or more enlarged arteries, feeding a serpiginous mass or nodule, and one or more draining veins (Fig. 4B and C) [5]. Contrast-enhanced magnetic resonance angiography (CEMRA) shows clearly all PAVMs (Fig. 4D) whose feeding arteries are larger than $3 \mathrm{~mm}$ [6]. Because PAVMs have predilections for affecting the lower lobes, exploration of the pleural sac is fundamental (Fig. 5A). Pulmonary angiography, which is an invasive procedure, less effective than chest $\mathrm{CT}$, is no longer used for diagnostic purposes and its use is restricted to treatment. Transarterial PAVM vasoocclusion with coils (Fig. 5B and C) is currently the treatment of choice [1]. Because of possible reperfusion more than the development of small PAVMs due to redistribution of the hemodynamic flow after transcatheter occlusion of large PAVMs, a CT or CE-MRA follow-up (Fig. 6A and B) is recommended [6]. Reperfusion of occluded PAVMs occurs in as many as 19\% [7] of them after initially successful treatment (Fig. 7A); the flux through the coils is better seen on CE-MRA (Fig. 7B) and is confirmed during the new vaso-occlusion procedure (Fig. 7C). Reperfusion risk increases with increasing PAVM feeding artery diameter, the use of a small number of coils (one or two) [8] (Fig. 8) or oversized coils, and coil placement proximally within the feeding artery. When reperfusion appears, the patient remains at risk for complications, so a new embolization must be performed [9]. When the cause is too proximal coil placement, the reperfusion can be due to a hypertrophy of a bronchial or systemic artery [10]; such a reperfusion does not cause shunting but can be responsible for hemoptysis.

\section{Brain}

The mechanisms of central nervous system (CNS) involvement in HHT are distal emboli, cerebrovascular malformation (CVM), and metabolic disorders [2, 11]. PAVM shunting that avoids the filtering effect of pulmonary capillaries is the most frequent mechanism inducing cerebral embolism and subsequent stroke (Fig. 9) or brain abscess secondary to cruoric and septic emboli, 
Fig. 2 A 57-year-old woman with a personal and familial (father, brother, and grandfather) history of epistaxis and multiples telangiectasia presented dyspnea leading to performance of a chest CT scan which discovered a unique pulmonary arteriovenous malformation (PAVM) of the left lower lobe. (A) Maximal intensity projection MDCT on the parasagittal plane shows a feeding artery (arrow), the aneurysm, and the draining vein (arrowhead) of a simple unique lower left lobe PAVM. (B) Anterior view of volumerendering technique MDCT of the same lower left lobe PAVM. (C) Movie 1: A $360^{\circ}$ view of the lower left PAVM. (D) Left lower lobe PAVM angiogram showing clearly the serpiginous architecture with one pulmonary artery (black arrow) feeding the PAVM and one draining vein (white arrow) without identification of an aneurysmal sac. (E) Movie 2: Dynamic angiogram of this PAVM shows the dynamic circulation of the iodine contrast
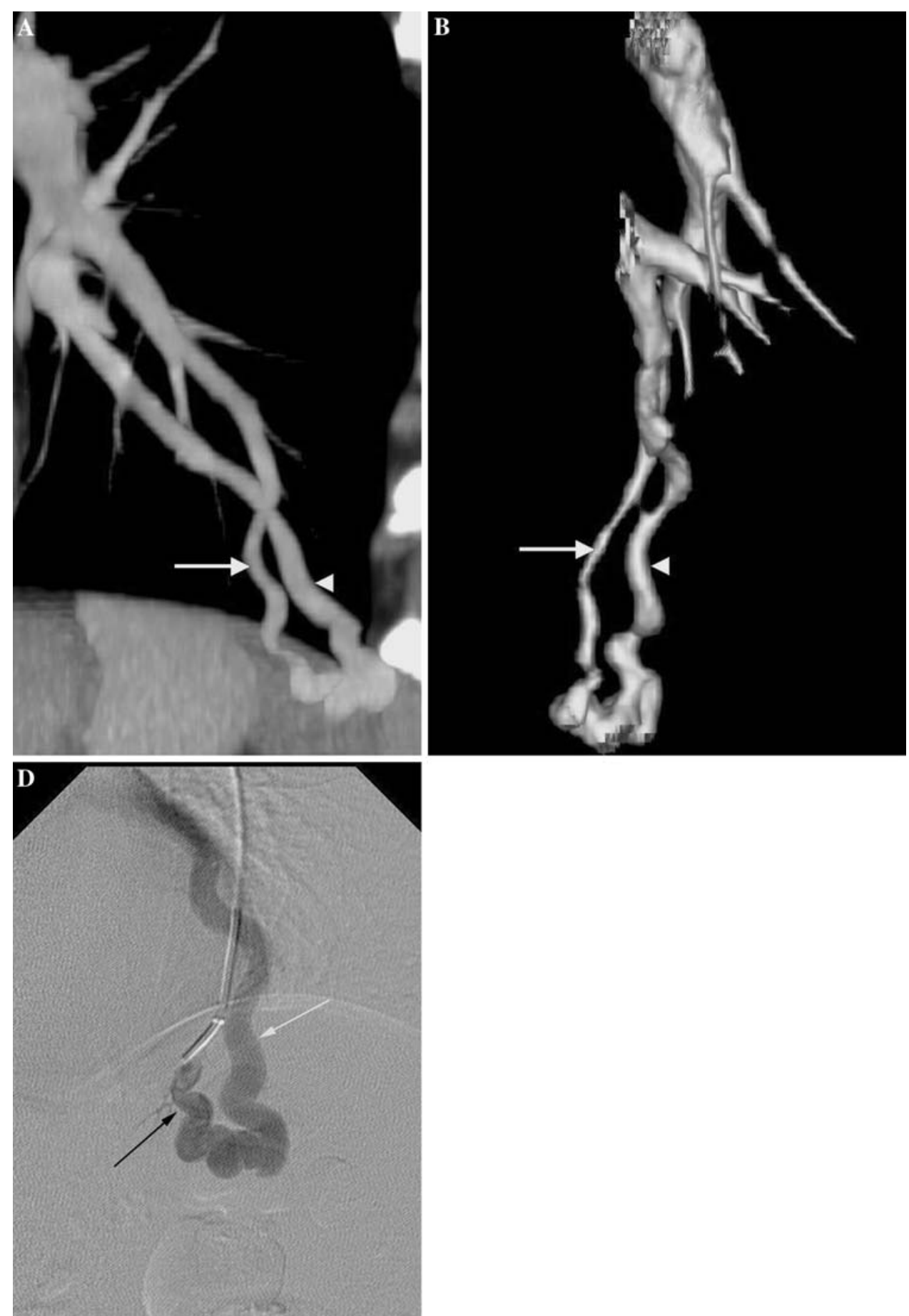

respectively (Fig. 10A). Furthermore, polycythemia secondary to chronic hypoxemia favors in situ formation of a cruoric embolus [1]. Multiple ischemic strokes in different arterial territories at different ages characterize the embolic mechanism in HHT (Fig. 9). Brain abscess is the most serious neurologic complication, occurring in 5\% to $10 \%$ of patients with PAVM. Typically in HHT these abscesses are multiple and recurrent, affecting the superficial layers of the cerebral lobes (mostly the parietal lobe) and developing often between the third and the fifth decade of life, when PAVMs increase in size and number. Transarterial PAVM vaso-occlusion is a key procedure to prevent these cerebral complications in HHT.

Spinal and cerebral vascular malformations are manifestations of underlying vascular dysplasia [12]. These lesions represent abnormal arteriovenous connections that failed to differentiate properly into arteriolar, capillary, and venular channels [13]. On magnetic resonance imaging 


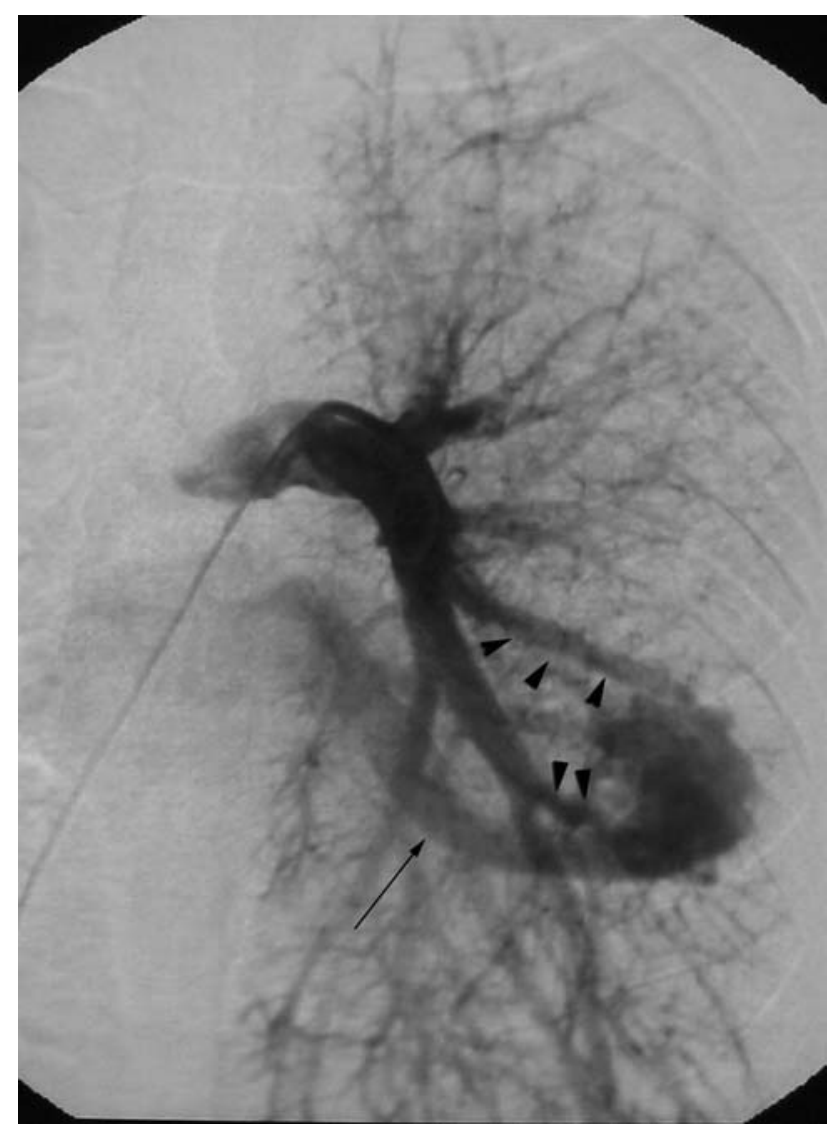

Fig. 3 A 23-year-old woman with a personal and familial history of HHT, in particular, a cerebral arteriovenous malformation in her grandmother, was sent for occlusion of multiple PAVMs including a complex malformation with two different segmental (arrowheads) arteries (lingular and left ventral inferior arteries) feeding a large reticulated fistula drained by two segmental veins (arrow)

(MRI), CVMs appear as areas of serpiginous flow voids insinuating into the brain parenchyma (Fig. 11). Patients with HHT often have multiple malformations of various types, some of them with an atypical or indeterminate MRI appearance [14]. Cerebral angiography may be required for diagnosis of equivocal lesions and for planning of treatment. The therapy for symptomatic CVMs is surgical resection, stereotactic radiosurgery, embolization, or a combination of these treatments [15].

Finally, the T1-weighted image hyperintensity of the basal nuclei correlates with a metabolic disorder related to hepatic portal vein shunt [16].

\section{Liver}

Hepatic involvement occurs in $30 \%$ to $73 \%$ of patients with HHT; most of them are asymptomatic or have a slight elevation of $\gamma$-glutamyltransferase (GGT) but rare complications occur, especially in female patients $[11,17]$.
Three different and often concurrent types of intrahepatic shunts (hepatic artery to hepatic veins, hepatic artery to portal vein, and portal vein to hepatic veins) are responsible for the parenchymal, vascular, and bile duct signs [11]. Hepatic abnormalities are diagnosed by Doppler ultrasound, MDCT, MRI, and MRA. Hepatic ultrasound associated with Doppler is a good tool with an experienced investigator [18]; otherwise findings can be considered as normal in the early stage because telangiectases are difficult to diagnose. The diameter of the common hepatic artery (>7 mm; Fig. 12A) and intrahepatic hypervascularization are suitable sonographic diagnostic parameters of HHT with a high sensitivity and specificity [18]. Rarely, Doppler ultrasound can reveal pulsatile portal flow (arterioportal shunt; Fig. 12B) or pulsatile hepatic vein flow (arteriovenous shunt; Fig. 12C).

Hepatic vascular lesions on MDCT range from tiny telangiectases (Fig. 12D) to transient perfusion abnormalities and large confluent focal vascular masses [19]. These lesions are often associated with arteriovenous, arterioportal, or portovenous shunts and hepatic artery enlargement (Fig. 12E-G). Telengiectases are hypervascular rounded nodules (varying from a few millimeters to $1 \mathrm{~cm}$ in size) localized in a subcapsular position in the arterial and late arterial phases, often becoming isointense with the hepatic parenchyma in the hepatic parenchymal phase. Arteriography, too aggressive for a screening and diagnosis tool, could be the best method to characterize telangiectases.

The natural evolution of hepatic teangiectasia in MRO patients is toward intrahepatic vascular shunts [20]. If simple telangiectasis can rarely provoke a painful liver with a slight elevation of GGT, arteriovenous shunts are more communicative. An arterioportal shunt may rarely induce portal hypertension, ascites, encephalopathy, and hematemesis. An arteriovenous shunt can, on rare occasions, be responsible for high-output heart failure and necrotizing cholangitis by arterial flow steal (the arterial supply of the biliary duct is exclusively dependent on the hepatic artery) [20]. Livers in patients with HHT may show either diffuse or partial hepatocellular regenerative activity, leading to regenerative focal nodular hyperplasia ( $\mathrm{FNH}$; Fig. 13A-D). The prevalence of FNH in patients with HHT is 100-fold greater than in the general population and can lead to dangerous hepatic biopsies. The combination of fibrosis (around abnormal vessels), nodular regenerative hyperplasia, and portal hypertension may lead to a misdiagnosis of cirrhosis [11]. Occasionally, patients with HHT present with true cirrhosis (secondary to extensive necrotizing cholangitis), hepatocarcinoma, or hemobilia (secondary to a MAV rupture in the biliary system). Angiomas can also coexist with telangiectases and hepatic MAV. Rarely, pancreatic telangiectasis can be associated with liver lesions (Fig. 14). 
Fig. 4 A 45-year-old man with a personal and familial (father, sister, and daughter) history of epistaxis and multiple telangiectasia presented pulmonary nodules on a chest X-ray. Unenhanced MDCT shows multiple PAVMs; rightto-left shunting was calculated as $17 \%$. (A) Chest $\mathrm{X}$-ray shows two well-defined nodules (arrows) with branching vessels (arrowheads). The third one is doubtful (large white arrow). (B) MDCT coronal 30-mmthick slab maximal intensity projection confirms the vascular structure of the nodules with branching vessels identified on the chest X-ray. Furthermore, it confirms the lower left PAVM (arrow) and shows a small right lower lobe PAVM not identified by chest X-ray (arrowhead). (C) MDCT axial 20-mm-thick slab maximal intensity projection shows clearly three PAVMs on the extreme right lower lobe (stars). These PAVMs are not visible on the chest X-ray. (D) Coronal CE-MRA shows the anatomy of the right upper lobe PAVM seen on the chest X-ray, with the feeding pulmonary artery (white arrowheads), the aneurysmal sac (arrow), and the draining vein (black arrowheads)
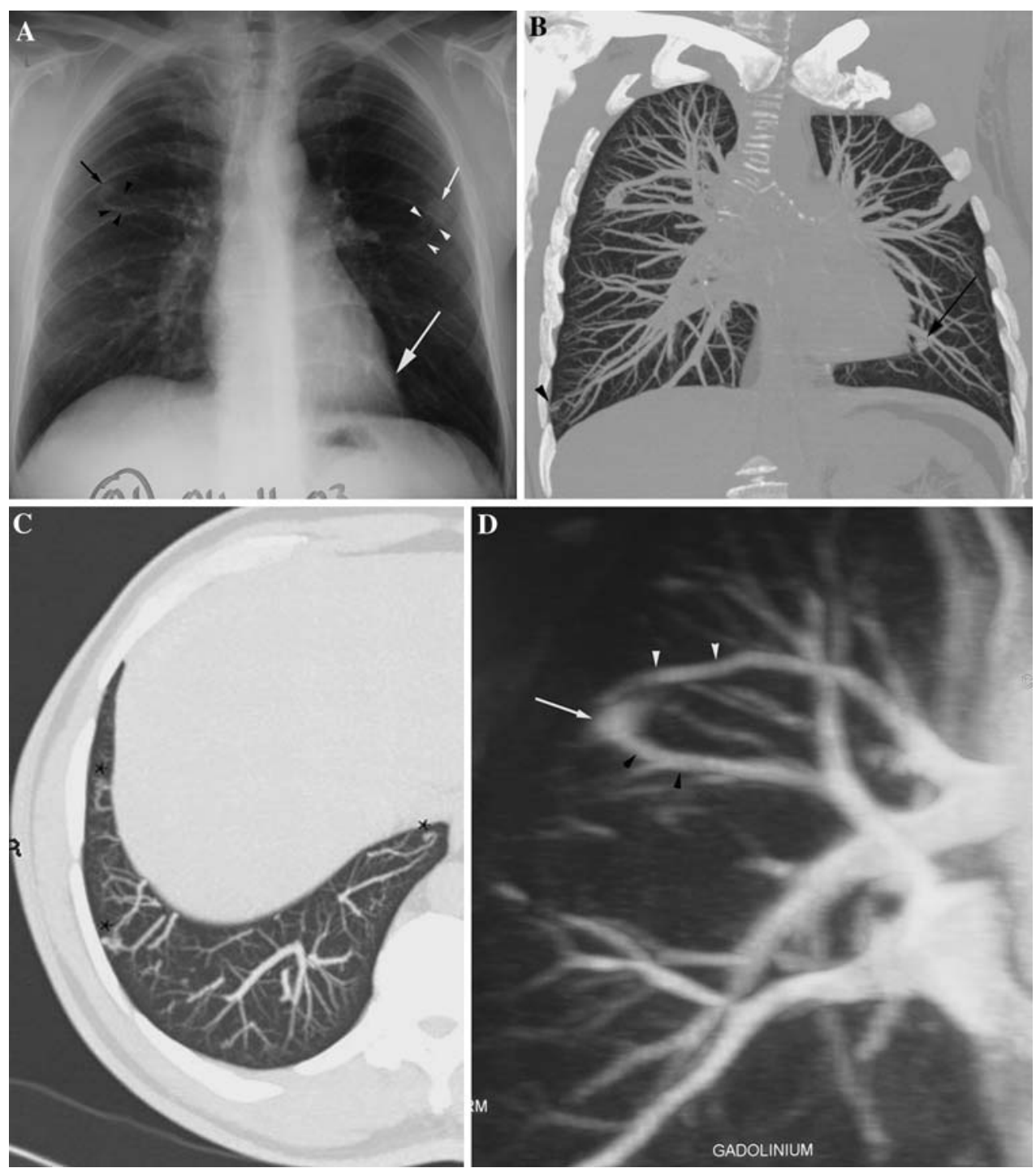

Transarterial embolization of liver arteriovenous fistulas has been performed but fatal outcomes due to necrotizing cholangitis suggest caution in its use. Orthotopic liver transplantation may represent the only definitive curative option for hepatic vascular malformations in HHT, even though at this time relapse has been observed in 2 of 28 patients undergoing transplantation [17].

\section{Gastrointestinal Tract}

Recurrent upper or lower gastrointestinal bleeding occurs in approximately $20 \%$ of patients with HHT and is responsible for anemia. The risk of fatal bleeding is mostly due to intestinal mucosal telangiectasis predominating in the gastroduodenal region, and less, frequently to intestinal $\mathrm{AV}$ shunt or angiodysplasia.
Classical imaging techniques are limited. Only large AV malformations can be diagnosed on CT scan. The technique of capsule endoscopy is emerging to study the small bowel. Endoscopy is the gold standard for diagnosis and therapy of gastroduodenal and colonic telangiectases [21].

\section{Summary}

In summary, knowledge of genetic findings and of phenotypes leads to optimal management of the patient and family. In these patients, diagnosis and adequate therapeutic choice, with a multidisciplinary approach, can prevent serious complications and avoid the need for hepatic biopsies in the case of hepatic involvement. 
Fig. 5 A 27-year-old woman with epistaxis since the age of 5 years, cutaneomucous telangiectasis, PAVMs, and a confirmed family history of HHT (mother, grandmother, and aunt). Three PAVMs were occluded at the age of 12 years; because of their small size, several PAVMs were not occluded at that time. Recently, the patient was admitted for ischemic stroke; the unenhanced MDCT shows clearly the enlargement of the previous PAVMs not treated. Right-toleft shunting is calculated as $15 \%$. (A) Coronal threedimensional volume-rendered image shows multiple lower lobe PAVMs without a PAVM on the upper lobes. Note the absence of the aneurysmal sac distally to the coil (white arrow) placed on the feeding artery of the PAVM 15 years ago. (B) Superselective angiogram of a right lower lobe PAVM shows clearly the angio-architecture of the fistulae. (C) Superselective angiogram control after transcatheter occlusion by a Nester coils (14 cm long, $4 \mathrm{~mm}$ diameter; Cook, Paris, France) showing the complete exclusion of the aneurysmal sac. The coil was placed as distally as possible and very compact to prevent reperfusion (arrow)
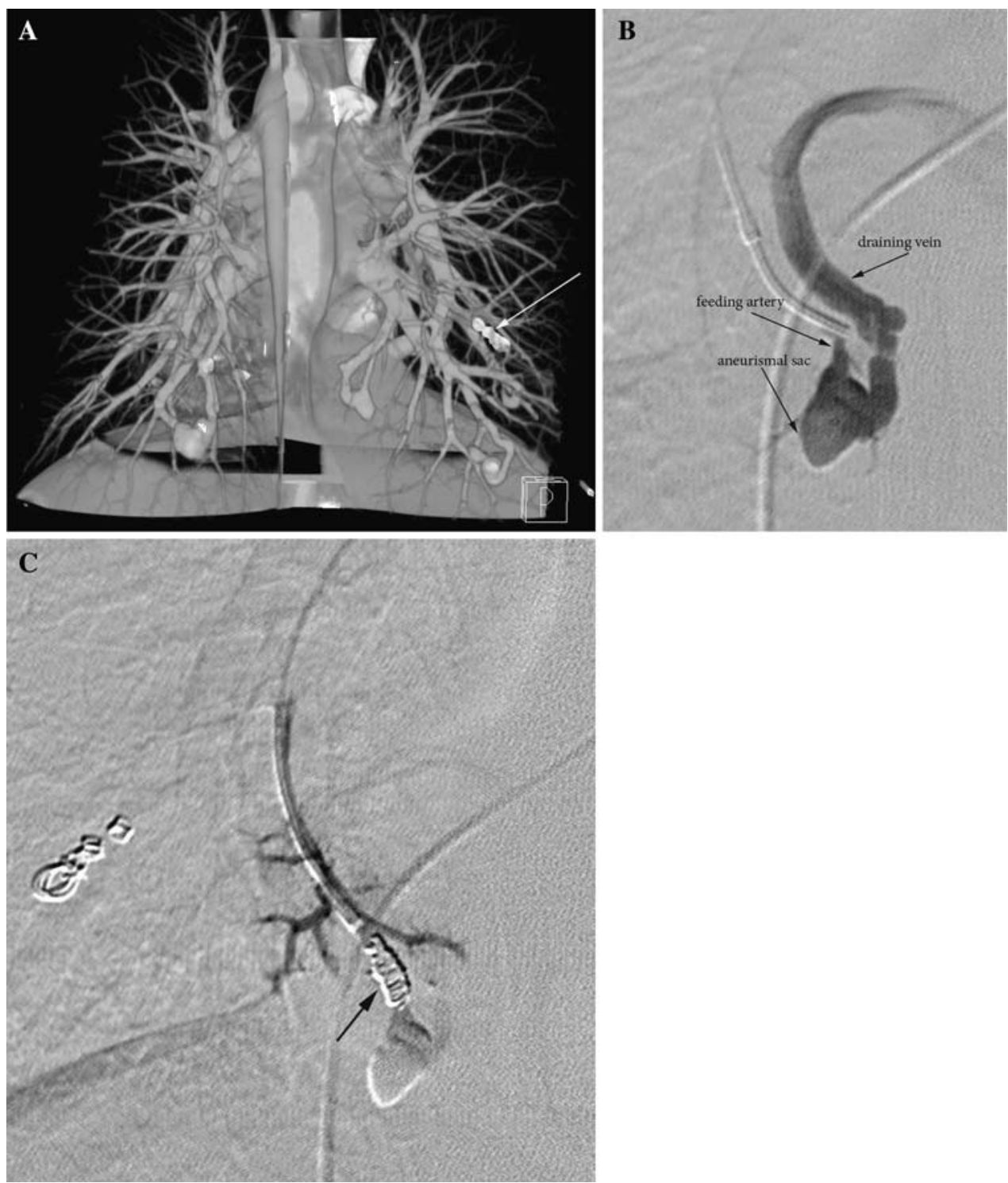

Open Access This article is distributed under the terms of the Creative Commons Attribution Noncommercial License which permits any noncommercial use, distribution, and reproduction in any medium, provided the original author(s) and source are credited.

\section{References}

1. Cottin V, Chinet T, Lavole A et al (2007) Pulmonary arteriovenous malformations in hereditary hemorrhagic telangiectasia: a series of 126 patients. Medicine (Baltimore) 86:1-17

2. Lesca G, Olivieri C, Burnichon N et al (2007) Genotype-phenotype correlations in hereditary hemorrhagic telangiectasia: data from the French-Italian HHT network. Genet Med 9:14-22

3. Todo K, Moriwaki H, Higashi M, Kimura K, Naritomi H (2004) A small pulmonary arteriovenous malformation as a cause of recurrent brain embolism. Am J Neuroradiol 25:428-430
4. White RI Jr, Lynch-Nyhan A, Terry P et al (1988) Pulmonary arteriovenous malformations: techniques and long-term outcome of embolotherapy. Radiology 169:663-669

5. Remy J, Remy-Jardin M, Wattinne L, Deffontaines C (1992) Pulmonary arteriovenous malformations: evaluation with CT of the chest before and after treatment. Radiology 182:809-816

6. Khalil A, Farres MT, Mangiapan G, Tassart M, Bigot JM, Carette MF (2000) Pulmonary arteriovenous malformations. Chest 117:1399-1403

7. Remy-Jardin M, Dumont P, Brillet PY, Dupuis P, Duhamel A, Remy J (2006) Pulmonary arteriovenous malformations treated with embolotherapy: helical CT evaluation of long-term effectiveness after 2-21-year follow-up. Radiology 239:576-585

8. Prasad V, Chan RP, Faughnan ME (2004) Embolotherapy of pulmonary arteriovenous malformations: efficacy of platinum versus stainless steel coils. J Vasc Interv Radiol 15:153-160

9. Milic A, Chan RP, Cohen JH, Faughnan ME (2005) Reperfusion of pulmonary arteriovenous malformations after embolotherapy. $\mathrm{J}$ Vasc Interv Radiol 16:1675-1683 
Fig. 6 A 24-year-old asymptomatic woman underwent routine chest radiography that showed multiple parenchymal vascular opacities. She fulfills the Curacao criteria for HHT. The biggest one was in the left lung. (A) A frontal view of the left selective pulmonary artery angiogram shows the large fistulae without clear evidence of other PAVMs. (B) A lateral left pulmonary artery angiogram view post transcatheter occlusion of the large PAVM (arrow) reveals several smaller but clinically significant (pulmonary artery diameters, $>3 \mathrm{~mm}$ ) fistulae in the anterior and subpleural space

(arrowheads). Both series were performed using the same rate $(20 \mathrm{ml} / \mathrm{s})$ and contrast volume $(40 \mathrm{ml})$
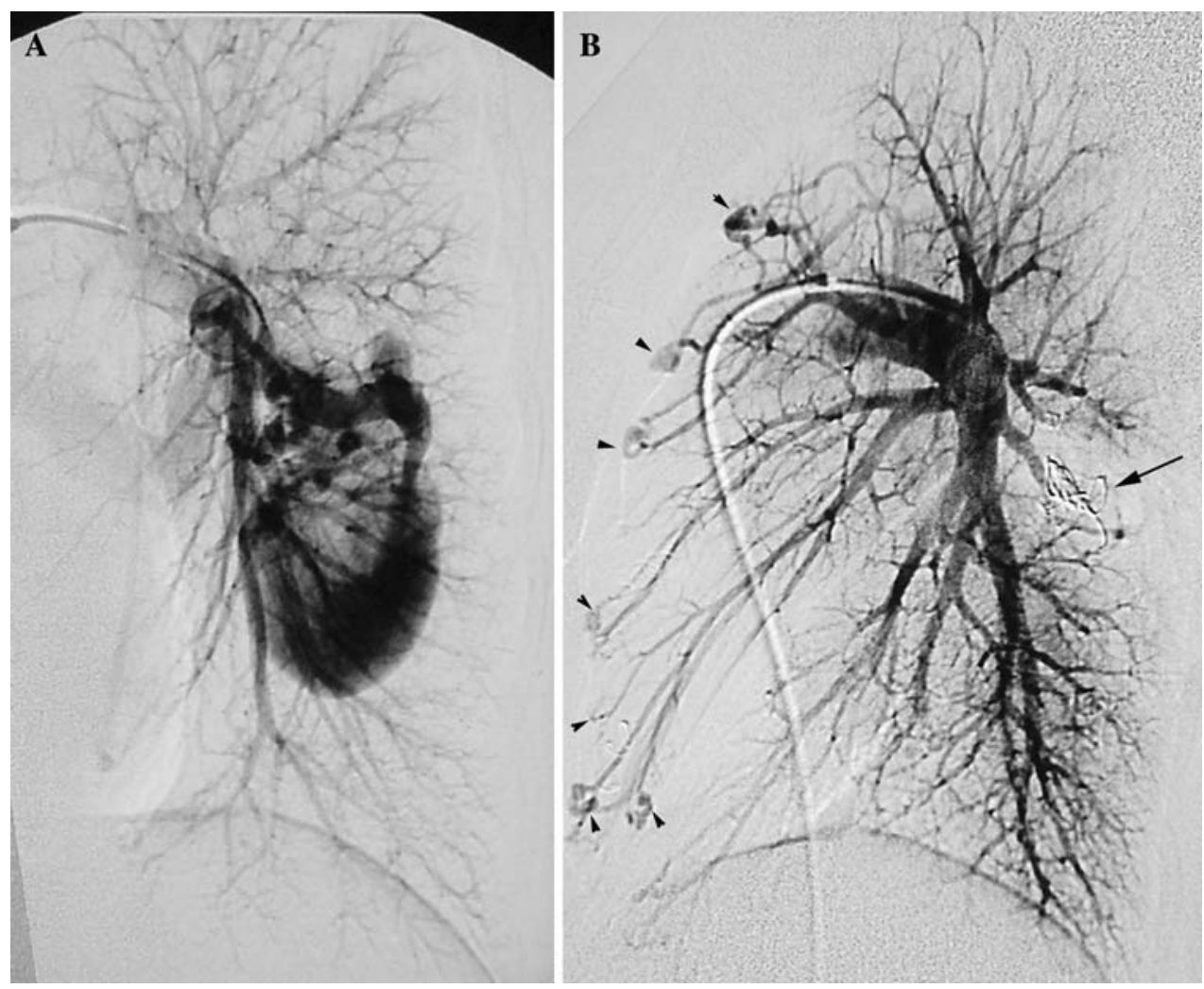

10. Brillet PY, Dumont P, Bouaziz N et al (2007) Pulmonary arteriovenous malformation treated with embolotherapy: systemic collateral supply at multidetector CT angiography after 2-20-year follow-up. Radiology 242:267-276

11. Buscarini E, Danesino C, Olivieri C, Lupinacci G, Zambelli A (2005) Liver involvement in hereditary haemorrhagic telangiectasia or Rendu-Osler-Weber disease. Dig Liver Dis 37:635-645

12. Rodesch G, Hurth M, Alvarez H, Tadie M, Lasjaunias P (2002) Classification of spinal cord arteriovenous shunts: proposal for a reappraisal-the Bicetre experience with 155 consecutive patients treated between 1981 and 1999. Neurosurgery 51:374379; discussion 379-380

13. Putman CM, Chaloupka JC, Fulbright RK, Awad IA, White RI Jr, Fayad PB (1996) Exceptional multiplicity of cerebral arteriovenous malformations associated with hereditary hemorrhagic telangiectasia (Osler-Weber-Rendu syndrome). Am J Neuroradiol 17:1733-1742

14. Maher CO, Piepgras DG, Brown RD Jr, Friedman JA, Pollock BE (2001) Cerebrovascular manifestations in 321 cases of hereditary hemorrhagic telangiectasia. Stroke 32:877-882

15. Maarouf M, Runge M, Kocher $M$, Zahringer M, Treuer $H$, Sturm V (2004) Radiosurgery for cerebral arteriovenous malformations in hereditary hemorrhagic telangiectasia. Neurology 63:367-369

16. Carette MF, Brugieres P, Khalil A (2007) Hyperintense basal ganglia on T1-weighted images in Rendu-Osler-Weber disease. Presse Med 36:883-884

17. Scelzo C, Greco S, Bonanni L et al (2007) The role of liver transplantation in the treatment of hereditary hemorrhagic telangiectasia: a short literature review. Transplant Proc 39:20452047

18. Caselitz M, Bahr MJ, Bleck JS et al (2003) Sonographic criteria for the diagnosis of hepatic involvement in hereditary hemorrhagic telangiectasia (HHT). Hepatology 37:1139-1146

19. Siddiki H, Doherty MG, Fletcher JG et al (2008) Abdominal findings in hereditary hemorrhagic telangiectasia: pictorial essay on 2D and 3D findings with isotropic multiphase CT. Radiographics 28:171-184

20. Garcia-Tsao G (2007) Liver involvement in hereditary hemorrhagic telangiectasia (HHT). J Hepatol 46:499-507

21. Longacre AV, Gross CP, Gallitelli M, Henderson KJ, White RI Jr, Proctor DD (2003) Diagnosis and management of gastrointestinal bleeding in patients with hereditary hemorrhagic telangiectasia. Am J Gastroenterol 98:59-65 


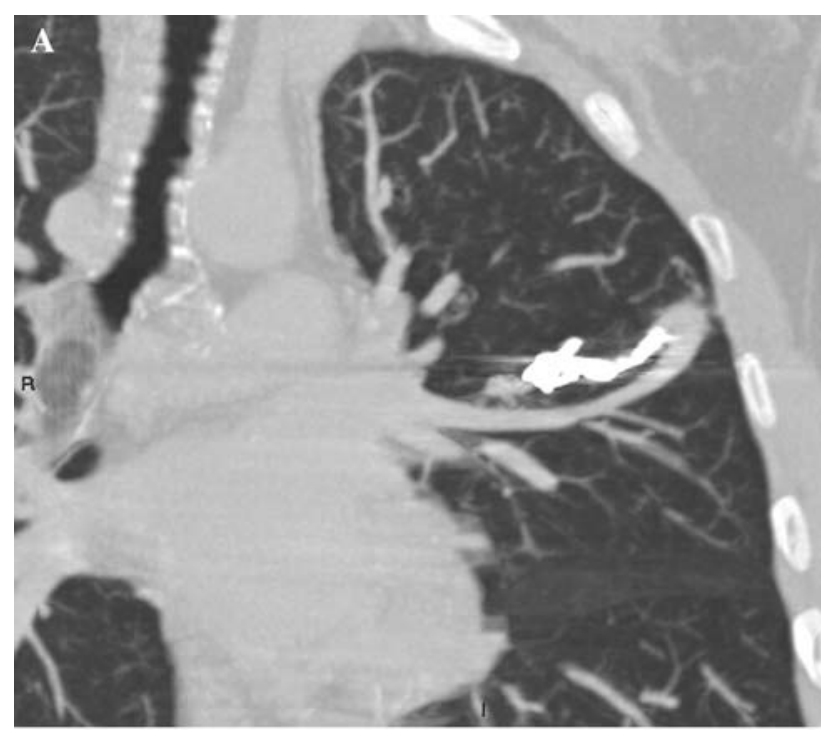

4Fig. 7 Follow up of the 45-year-old patient described in the legend to Fig. 3. Coronal maximal intensity projection MDCT (A) and coronal contrast-enhanced MRA (B) show the reperfusion of a treated PAVM. The absence of coil artifact in CE-MRA gives a direct sign of reperfusion by visualizing the reperfused artery (arrowheads). The MDCT signs are indirect: persistence of the aneurysmal sac and of a large draining vein. (C) Standard angiography confirms the reperfusion. The elongation of the uncompacted coil and the proximal placement of the coil into the feeding artery were probably risk factors for reperfusion
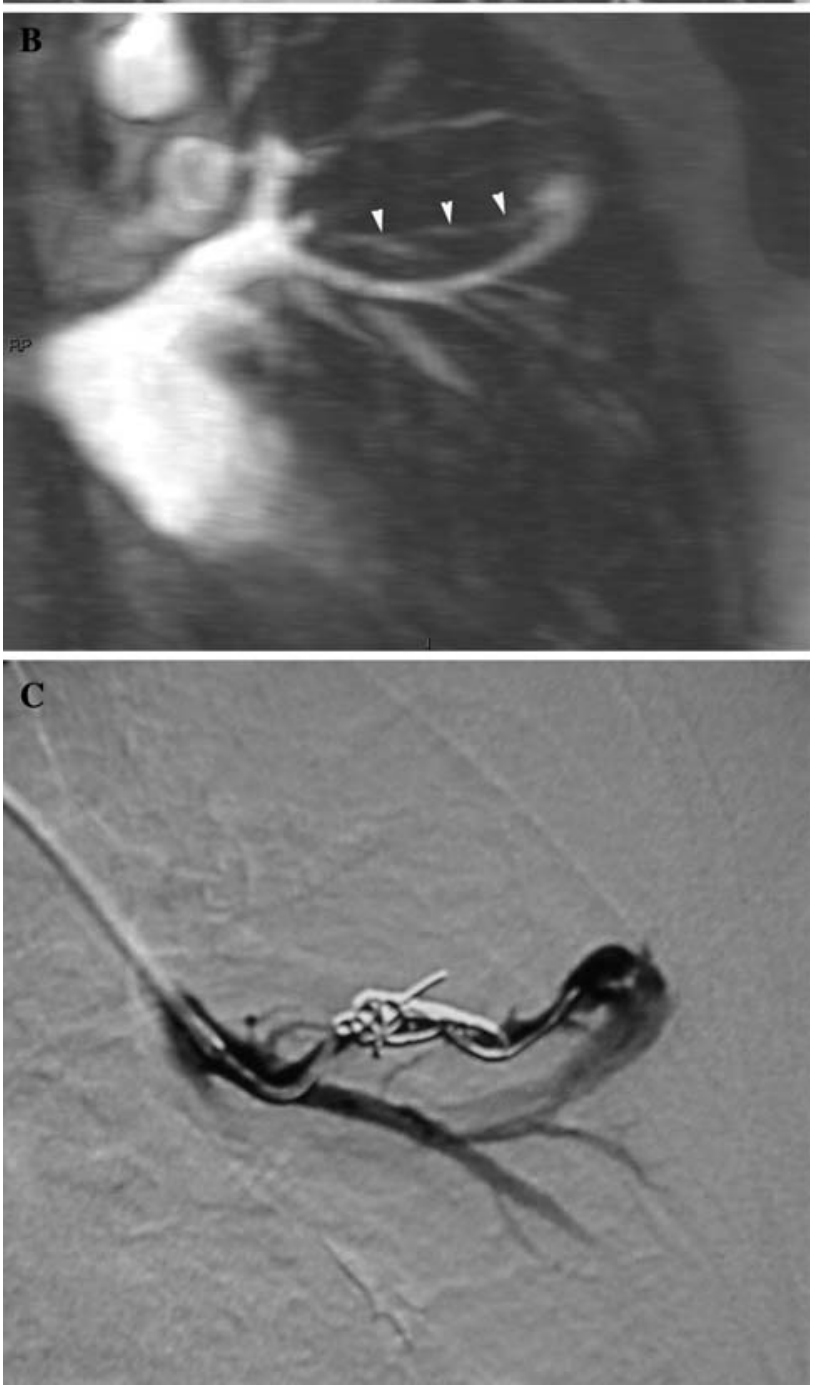


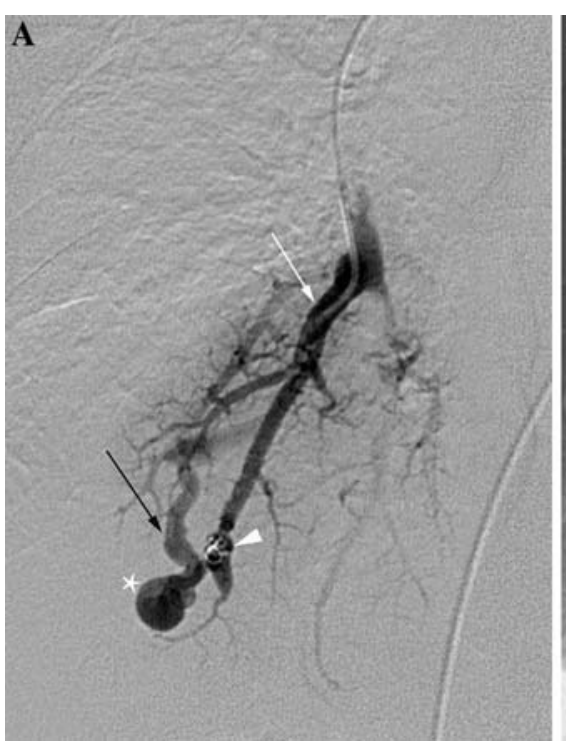

Fig. 8 A 77-year-old woman diagnosed with HHT with pulmonary involvement and AKL1 genotype, with a history of PAVM embolization and reperfusion, neurological complications, and important epistaxis was hospitalized for repeat transcatheter occlusion of a reperfused PAVM. (A) Superselective angiogram of the feeding artery (white arrow) shows the single coil (white arrowhead), the aneurysmal

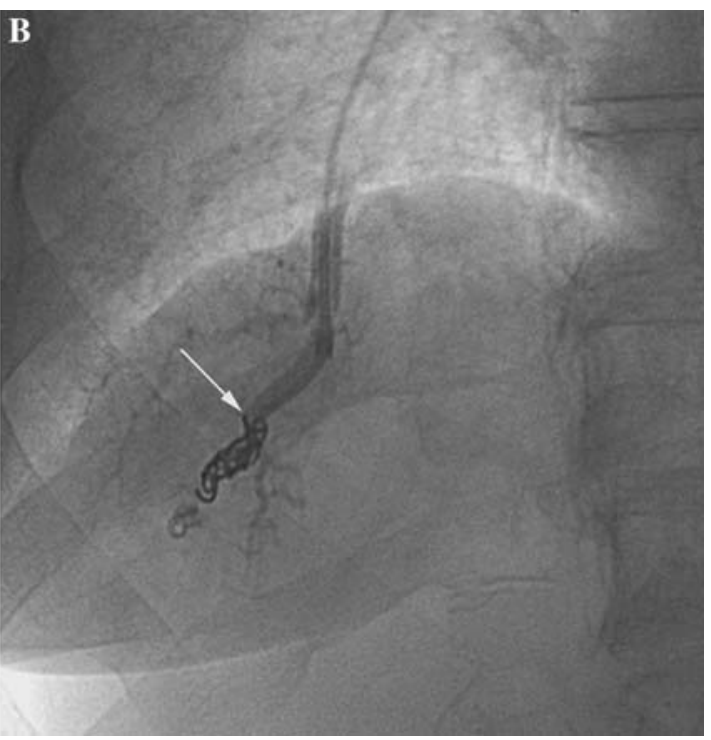

sac (asterisk), and the large draining vein (black arrow). (B) Superselective angiogram control of the PAVM shows complete occlusion of the feeding artery (white arrow) and the aneurysm. Although, to prevent reperfusion, the first single coil was placed distally, right next to the aneurysmal sac, reperfusion occurred. The use of a single coil is a risk factor for reperfusion
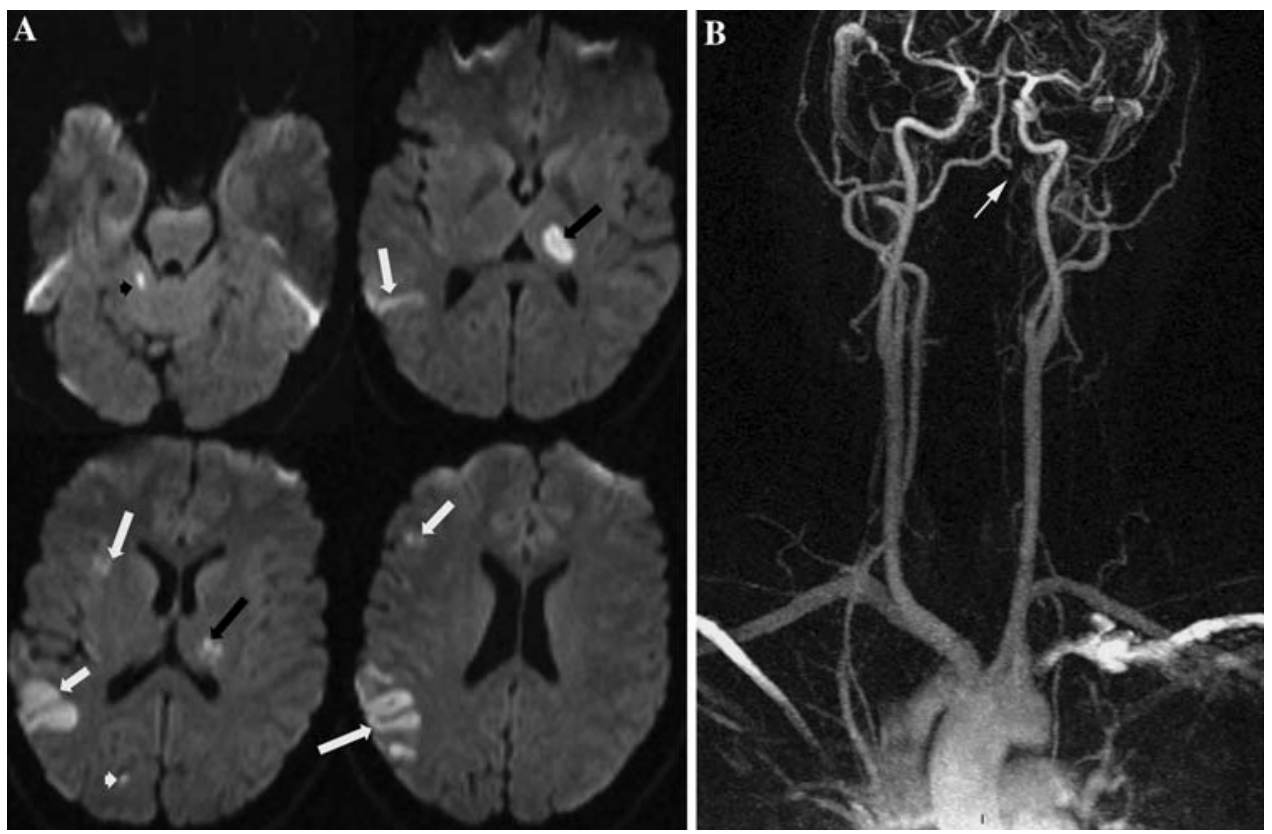

Fig. 9 A 36-year-old woman presenting with multiple acute cerebral infarctions. The intracerebral and cervical vessel checkup was normal except for the left vertebral artery occlusion. The cardiac evaluation and chest CT scan show a right-to-left shunt by a large PAVM of the internal segment of the right middle lobe. The genetic study was positive for ALK1, but it is not formally recognized as a pathogen by geneticists. (A) Serial images of diffusion weighted images (diffusion $b$ value $=1000 \mathrm{~s} / \mathrm{mm}^{2}$ ) shows multiple cerebral infarction involving the right middle cerebral artery (white arrows), the right (white arrowheads) and left (black arrows) posterior cerebral arteries, and the right superior cerebella artery (black arrowheads) territories. (B) CE-MRA of the neck vessels shows a left vertebral artery occlusion (arrow), potentially to cruoric emboli 


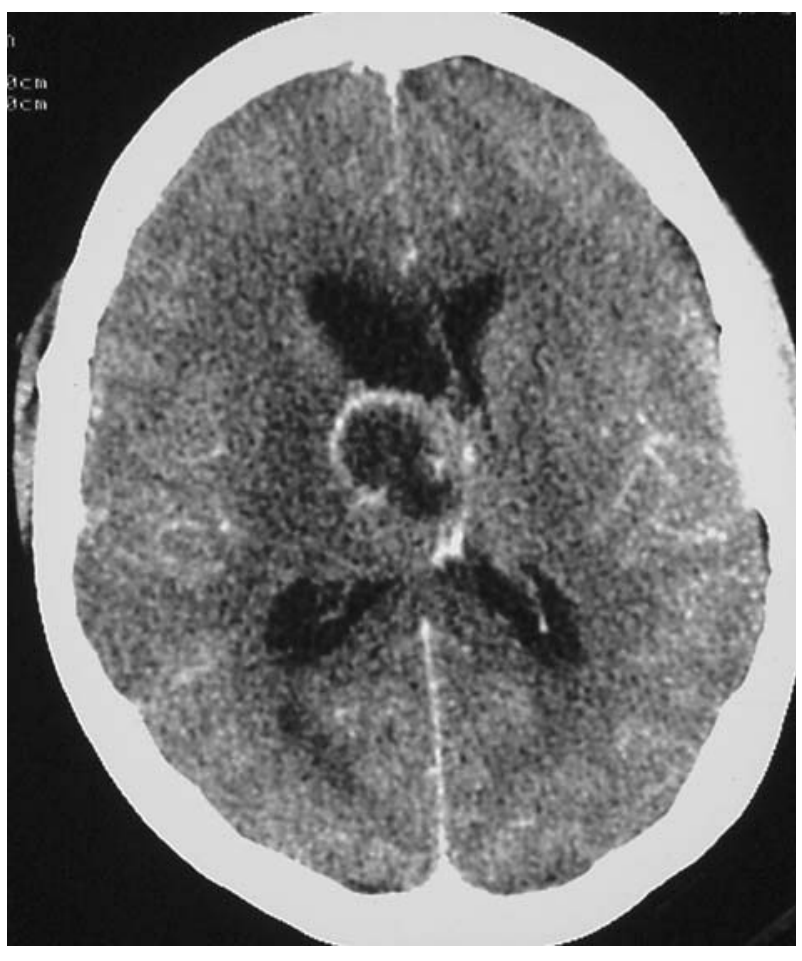

Fig. 10 A 25-year-old woman presenting with headache and fever. Enhanced CT scan shows a ring contrast enhancement mass of the right basal ganglia in association with a brain abscess. The etiological research revealed multiple PAVMs and mucocutaneous telangiectasis consistent with HHT

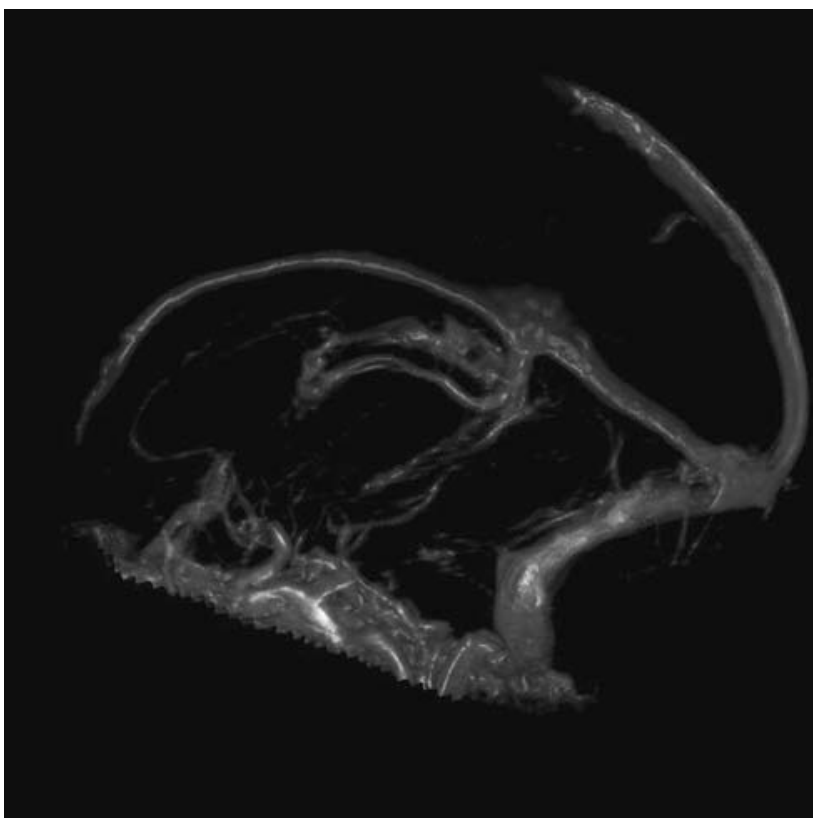

Fig. 11 A 55-year-old woman with HHT presenting important epistaxis inducing anemia, multiple telangiectasis, and liver and pancreatic involvement without pulmonary abnormalities. She had brain MR angiography for systematic evaluation, which shows a small cebrovascular malformation (arrow): follow-up and no endovascular treatment were decided 
Fig. 12 Hepatic abnormalities in several patients with HHT. (A) Hepatic artery (A) enlargement at $9 \mathrm{~mm}$ on ultrasound (US). (B) Doppler US of the right portal branch shows pulsatile portal flow consistent with an hepatic artery-to-portal vein shunt (arterioportal shunt). (C) Doppler US of the left hepatic vein shows pulsatile flow into the left hepatic vein consistent with an hepatic artery-to-hepatic vein shunt (arterioveinous shunt). (D) A 2-mm-thick coronal reformatting MDCT during arterial phase shows hepatic telangiectasis (arrows).

(E) Axial, (F) sagittal, and (G) coronal maximal intensity projection MDCT reformatting with a 15 -mm slice shows major hepatic artery hypertrophy with arterial calcifications (black arrow) and celiac trunk hypertrophy in a patient with giant left basal PAVM (asterisk). Note the left inferior phrenic artery (white arrow) enlargement (in comparison with the right inferior phrenic artery; white arrowheads) to supply the flow steal of the left lower lobe pulmonary artery by the giant PAVM, inducing relative lower lobe ischemia
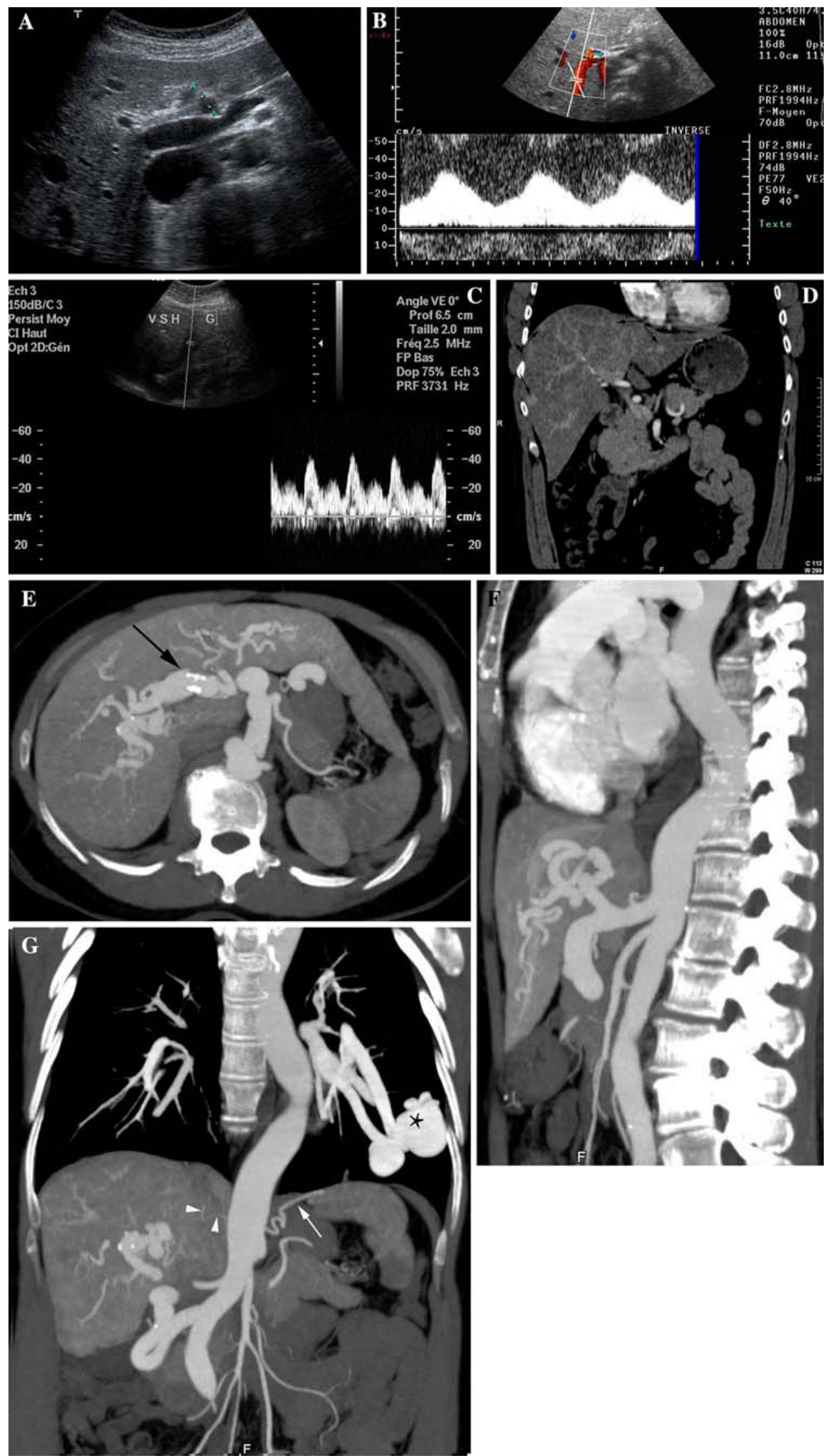

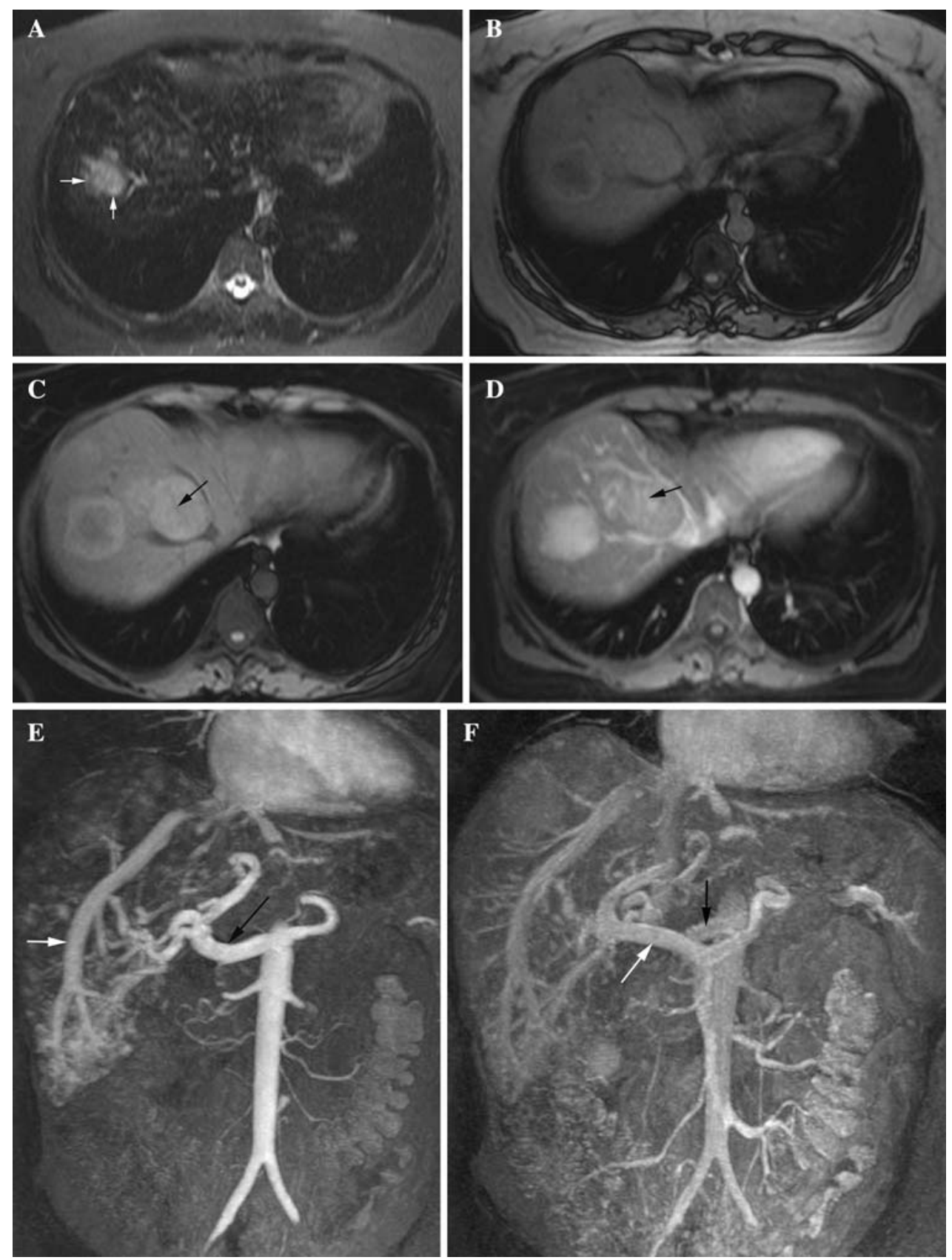

Fig. 13 Same patient as in Fig. 12D. Hepatic abnormalities explored by liver MRI: liver pseudotumor and vascular malformations. (A) Axial TSE T2 short TI inversion recovery (TSE-STIR) image shows a hyperintense mass involving segment VII (arrow) without any abnormalities in segment VIII. (B) Axial T1-weighted fast low-angle shot (FLASH) image shows the hyper signal ring of the segment VII lesion and isointensity relative to the normal hepatic tissue signal mass of segment VIII. (C) Axial fat-suppressed T1-weighted FLASH image shows a homogeneous bright lesion with hypointense central scar (black arrow) of the segment VIII lesion and the highly intense ring of the segment VII lesion. (D) Axial delayed contrast-enhanced T1-weighted FLASH image shows that the segment VII lesion has homogeneous enhancement corresponding to vascular malformation, and the segment VIII lesion is isointense relative to normal hepatic tissue with enhancement of the central scar (black arrow) corresponding to focal nodular hyperplasia. (E) Coronal arterial-phase contrast-enhanced MR angiography shows hepatic artery enlargement (black arrow) and hepatic artery-to-hepatic vein shunting with hepatic vascular malformations. The rapid enhancement of the hepatic vein (right arrow) before portal vein and renal vein enhancement signal the hepatic artery-to-hepatic vein shunting within the hepatic vascular malformation. (F) Coronal portal-phase contrast-enhanced MRA shows that the portal vein (white arrow) is permeable and has the same dimension as the enlarged hepatic artery (black arrow). (G) Movie 3 shows the maximal intensity projection of arterial-phase contrast-enhanced MR angiography of the upper abdominal area 


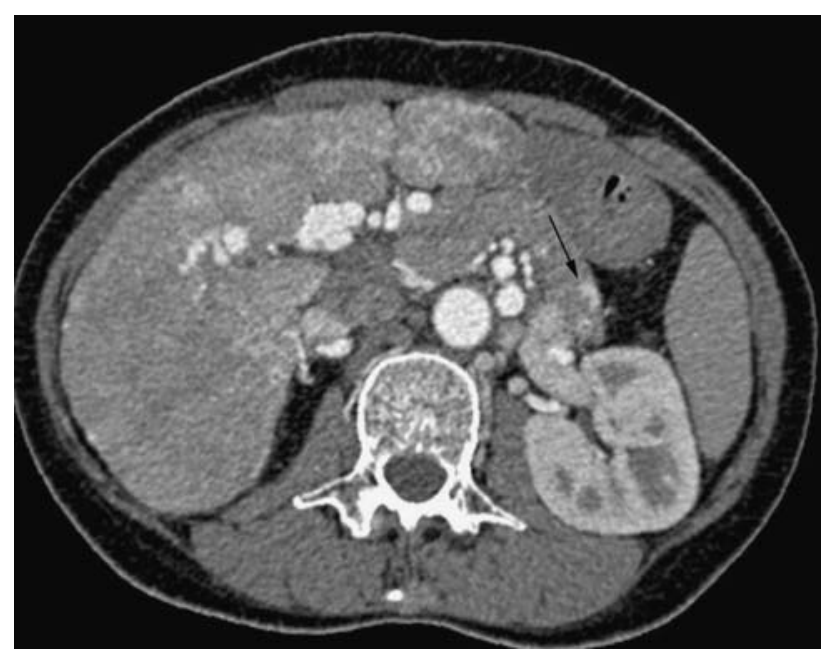

Fig. 14 A 55-year-old woman with a history of recurrent epistaxis, intestinal bleeding, and liver involvement of HHT. Abdominal contrast-enhanced MDCT shows hepatic and pancreatic abnormalities. A 1-mm-thick axial image in the arterial phase shows clearly the pancreatic telangiectasis (arrow), hepatic left lobe enhancement heterogenicity, and hepatic artery enlargement 